\title{
Integer quantum Hall effect on a six valley hydrogen-passivated silicon (111) surface
}

\author{
K. Eng, R. N. McFarland, and B. E. Kane \\ Laboratory for Physical Sciences, University of Maryland at College Park, College Park, MD 20740 USA
}

(Dated: July 13, 2018)

\begin{abstract}
We report magneto-transport studies of a two-dimensional electron system formed in an inversion layer at the interface between a hydrogen-passivated $\mathrm{Si}(111)$ surface and vacuum. Measurements in the integer quantum Hall regime demonstrate the expected sixfold valley degeneracy for these surfaces is broken, resulting in an unequal occupation of the six valleys and anisotropy in the resistance. We hypothesize the misorientation of $\mathrm{Si}$ surface breaks the valley states into three unequally spaced pairs, but the observation of odd filling factors, is difficult to reconcile with noninteracting electron theory.
\end{abstract}

PACS numbers: PAC Nos. 73.40.-c, 73.43.Qt, 71.70.Di

The silicon field effect transistors (FETs) that are at the heart of contemporary microelectronics rely on mobile electrons or holes confined at the interface between $\mathrm{Si}$ and a higher bandgap barrier material. This barrier in metal oxide silicon (MOS) FETs is $\mathrm{SiO}_{2}$, an amorphous material which introduces inevitable disorder at the $\mathrm{Si}-\mathrm{SiO}_{2}$ interface and limits the carrier mobility in these devices. A crystalline interface can be created using epitaxial SiGe-Si layers in which mobilities can be over an order of magnitude higher than the best MOSFET devices [1], but this technique is limited to the [100] oriented surfaces 2]. Recently a new technique for Si crystalline interfaces has been demonstrated [3] in which a $\mathrm{Si}$ interface is passivated with a monolayer of hydrogen and the barrier material is a vacuum. While the inertness and high degree of atomic perfection of these surfaces has been known for some time [4, 5, 6], the development of high mobility electronic devices on $\mathrm{H}-\mathrm{Si}$ enables the exploration of two-dimensional (2D) physics of novel Si surface orientations and may one day allow quantum devices to be engineered at the atomic scale using surface manipulation techniques [7].

We report here the first detailed magneto-transport studies of a 2D electron system (2DES) at a H-Si(111) surface gated through a vacuum barrier. Electron mobilities are an order of magnitude higher $\left(24,000 \mathrm{~cm}^{2} / \mathrm{Vs}\right)$ than $\mathrm{Si}(111)$ MOSFETs, enabling the observations of the integer quantum Hall effect (IQHE). In the effective mass approximation the ground state for a $2 \mathrm{DES}$ on the $\mathrm{Si}(111)$ surface is six-fold degenerate with each $\mathrm{Si}$ conduction band valley contributing an equal number of carriers, (Fig. 1d) each with anisotropic in-plane masses, $m_{x}=0.19 m_{o}$ and $m_{y}=0.67 m_{o}[8]\left(m_{o}\right.$ is the mass of the free electron). However, measurements of Shubnikovde Haas $(\mathrm{SdH})$ oscillations in $\mathrm{Si}(111)$ MOSFETs have shown conflicting valley degeneracies of two $8,[9,10]$ and six 11, 12] along with isotropic resistivities for both. Subsequent proposals [11, 13] have tried to explain these anomalous observations, but to date conclusive experimental results are still lacking.

The high mobility 2DES can be created by contact

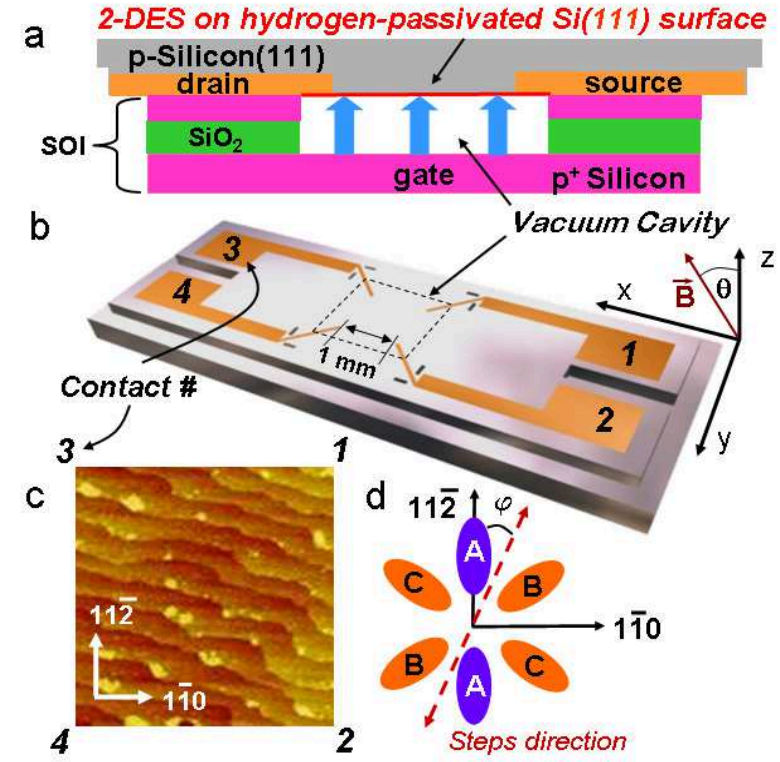

FIG. 1: (a) Schematic cross-section of a H-Si(111) substrate contact bonded to a SOI substrate. $\mathrm{A} \mathrm{p}^{+}$layer in the SOI defines the gate, where blue arrows depict the electric field. A 2DES is formed at the H-Si(111) surface within an encapsulated cavity. (b) The H-Si(111) substrate has four $\mathrm{n}^{+}$contacts numbered accordingly. Tilted magnetic fields are applied in the $x$ - $z$ plane. (c) A $1 \mu \mathrm{m} \times 1 \mu \mathrm{m}$ AFM image of atomic steps on a $\mathrm{H}-\mathrm{Si}(111)$ surface in relation to the crystal directions and the contacts of the device. (d) The projection of the six valleys for the $\mathrm{Si}(111)$ surface with pairs of valleys labeled $\mathrm{A}, \mathrm{B}$ and $\mathrm{C}$.

bonding two individual Si substrates[3] (Fig. 1a). One is the $\mathrm{H}-\mathrm{Si}(111)$ substrate (float zone, p-type, $\rho \sim 10 \Omega$-cm) which has four phosphorous contacts forming a 1-mmwide square with sides oriented parallel to the [110] and $[11 \overline{2}]$ crystallographic directions (Fig. 1b and 1c). The second is a silicon-on-insulator (SOI) substrate which acts as the remote gate, where an electric field can be controlled within an etched cavity. The $\mathrm{Si}(111)$ surface is H-passivated by immersion in an ammonium fluoride solution. The two substrates are then bonded in vacuum $\left(\sim 10^{-6}\right.$ Torr $)$, which allows the remote gate to induce 


\begin{tabular}{ccccc}
\hline \hline$\nu$ & $\mathrm{n}\left(\times 10^{11} \mathrm{~cm}^{-2}\right)$ & $\theta$ & $\Delta_{(13,24)}(\mathrm{K})$ & $\Delta_{(12,34)}(\mathrm{K})$ \\
\hline 2 & 5.69 & $0^{\circ}$ & $8 \pm 0.5$ & $7 \pm 0.5$ \\
3 & 6.50 & $39^{\circ}$ & $0.4 \pm 0.2$ & $0.8 \pm 0.2$ \\
4 & 6.50 & $48.4^{\circ}$ & $1.1 \pm 0.2$ & $1.2 \pm 0.2$ \\
6 & 6.50 & $48.4^{\circ}$ & $1.1 \pm 0.2$ & $1.2 \pm 0.2$ \\
\hline \hline
\end{tabular}

TABLE I: Energy gaps for various filling factors, where $\theta=0^{\circ}$ corresponds to a magnetic field normal to the 2DES. The subscripts in $\Delta_{(12,34)}$ represent the measurement orientation.

electrons on the $\mathrm{H}-\mathrm{Si}(111)$ surface and protects the air sensitive surface inside the cavity.

Figure 2a shows traces of the Hall $\left(R_{x y}\right)$ and the longitudinal resistance $\left(R_{x x}\right)$ as a function of perpendicular magnetic field, $B_{\perp}$, for a constant electron density, $n=6.5 \times 10^{11} \mathrm{~cm}^{-2}$ at $T=300 \mathrm{mK}$. We have obtained similar data in two devices over a wide range of $n$. We present data for a single device at a density where IQHE features are most apparent. There are two orientations in which $R_{x x}$ is measured; $R_{(12,34)}$ and $R_{(13,24)}$, where the first and second subscripts represent the current and voltage contacts respectively (Fig. 1b). Anisotropy in $R_{x x}$ is observed, but both orientations exhibit minima that occur at integer values of filling factor, $\nu=n h / e B_{\perp}$. Similar behavior (within $3 \%$ ) is also observed for $\mathrm{R}_{(43,21)}$ and $\mathrm{R}_{(42,31)}$. These minima are directly correlated with the observed plateaus in $R_{x y}$, a trademark of the QHE. Filling factors less than six are observed at $\nu=5,4$, 3 , and 2 , where $\nu=5$ is apparent through $d^{2} R_{x x} / d B_{\perp}^{2}$. Figure $2 \mathrm{~b}$ shows characteristic behavior at low $B$ fields: $R_{x y} / B_{\perp}$ is dependent on $B_{\perp}$; both $R_{(12,34)}$ and $R_{(13,24)}$ display a positive magneto-resistance (MR); finally, while more apparent in $R_{(12,34)}$, both orientations display $\mathrm{SdH}$ oscillations with minima occurring approximately every eighth Landau level (LL) filling factor, $\Delta \nu \sim 8$.

Classical MR calculations 14 show that in a multicomponent electron system in which electrons have differing anisotropic in-plane masses, $R_{x y}$ is non-linear with respect to $B_{\perp}$, and $R_{x x}$ should display a positive MR. In Figure $2 \mathrm{~b}$ we plot $R_{x y} / B_{\perp}$ and $R_{x x}(B)$ overlaid with a classical MR model in which all six valleys are equally occupied, with $n$ and the mean scattering time, $\tau$, as free parameters. The values which provided the best fit to $R_{x y}$ are within $5 \%$ of observed values: $\tau=3.5$ ps and $n \simeq 6.8 \times 10^{11} \mathrm{~cm}^{-2}$. This model agrees well with the observed behavior in $R_{x y}$ and the positive MR in $R_{x x}$. However, in order to explain the observed anisotropy in $R_{x x}$, an unequal population of electrons among the six valleys is required.

The strong $R_{x x}$ minimum at $\nu=2$, suggests a gap between the six valleys. We obtain gap energies, $\Delta_{\nu}$, from the temperature dependence of $R_{x x}, R_{x x} \sim$ $\exp \left(-\Delta_{\nu} / 2 k_{B} T\right)$. These measurements show that the energy gap, $\Delta_{2}$, is insensitive to small changes in $B_{\perp}$ and inplane fields, $B_{\|}$(Table 1$)$. It is by far the largest gap $(\simeq 7$ $\mathrm{K})$ and provides strong evidence that the six valleys are indeed broken asymmetrically following a 4-2 valley con-
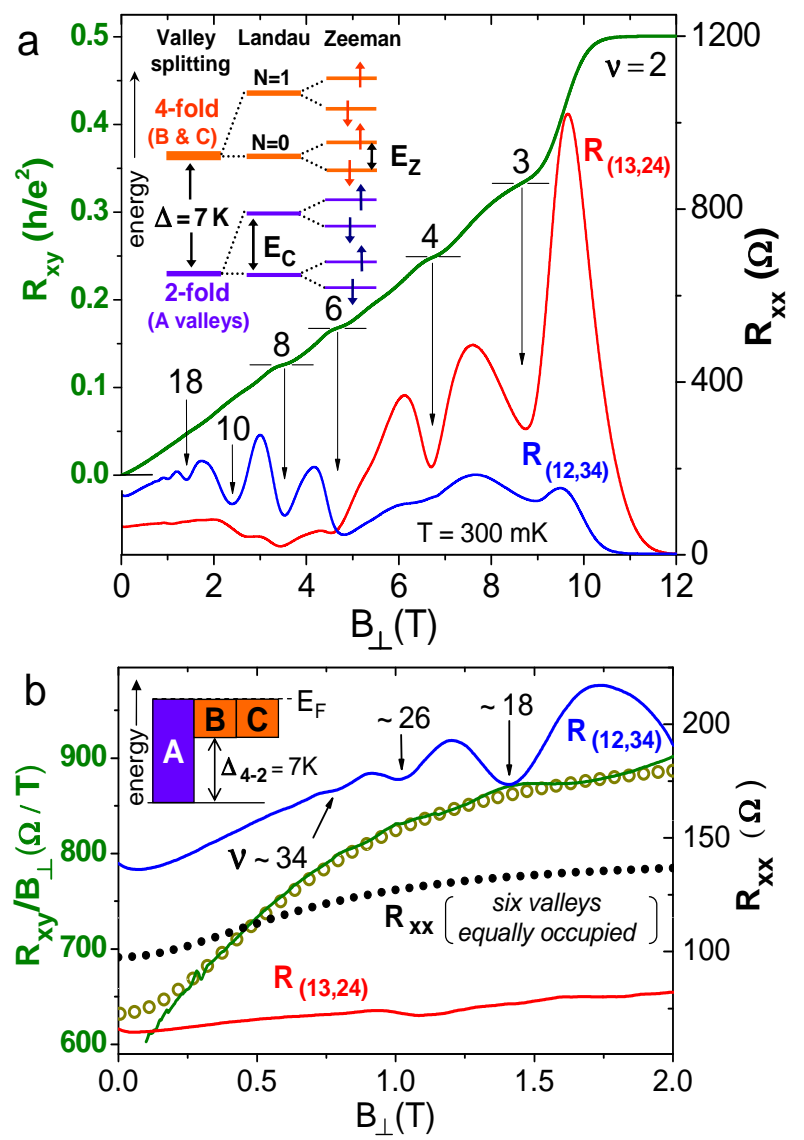

FIG. 2: (a) $R_{x y}$ (green), $R_{(12,34)}$ (blue) and $R_{(13,24)}$ (red) versus $B_{\perp}$ for the device at $T=300 \mathrm{mK}$ and $n=6.5 \times$ $10^{11} \mathrm{~cm}^{-2}$. Plateaus in $R_{x y}$ are labeled by horizontal bars and the numbers indicate their corresponding filling factor $\nu$. The inset is a schematic energy level diagram depicting the three energy scales affecting $\mathcal{D}(E)$ at $B_{\perp}=1 \mathrm{~T}$. (b) The same data from (a) is plotted within $0 \leq B_{\perp} \leq 2 \mathrm{~T}$. Hall resistance is plotted as $R_{x y} / B_{\perp}$ and the divergence is removed for $B_{\perp} \leq 0.1 \mathrm{~T}$. Open symbols $\left(R_{x y} / B_{\perp}\right)$ and closed symbols $\left(R_{x x}\right)$ correspond to calculations having all six valleys equally occupied. The inset depicts $\mathcal{D}(E)$ for the $7 \mathrm{~K}$ model at $B=0$.

figuration. For simplicity we will assume a model which is composed of only one type of splitting which is independent of $B$ : two valleys (A) are $7 \mathrm{~K}$ lower in energy than the remaining four valleys (B and $\mathrm{C}$ ) in the ground state (inset of Fig. 2b). The inset of Fig. 2a illustrates the effect $B_{\perp}$ has on the the density of states, $\mathcal{D}(E)$, for this ' 7 K model'. Quantization of the electron's orbital motion creates LLs which are separated by the cyclotron gap, $E_{C}=\hbar e B_{\perp} / m_{\mathcal{D}}$, where $m_{\mathcal{D}}=\left(m_{x} m_{y}\right)^{1 / 2}=0.358 m_{o}$ is the density of states mass for $\mathrm{Si}(111)$. Each LL then splits into two energy levels due to spin. This energy separation is the Zeeman energy, $E_{Z}=g^{*} \mu_{B} B_{\perp} / \cos \theta$, where $g^{*}$ is the effective g-factor and $\mu_{B}$ is the Bohr magneton.

The validity of this $7 \mathrm{~K}$ model can be tested with measurements in $R_{x x}$ versus tilted $B$ fields. Rotating the device some angle, $\theta$, away from $B_{\perp}$ changes $E_{Z}$ with respect to $E_{C}$, and eventually energy levels of different 


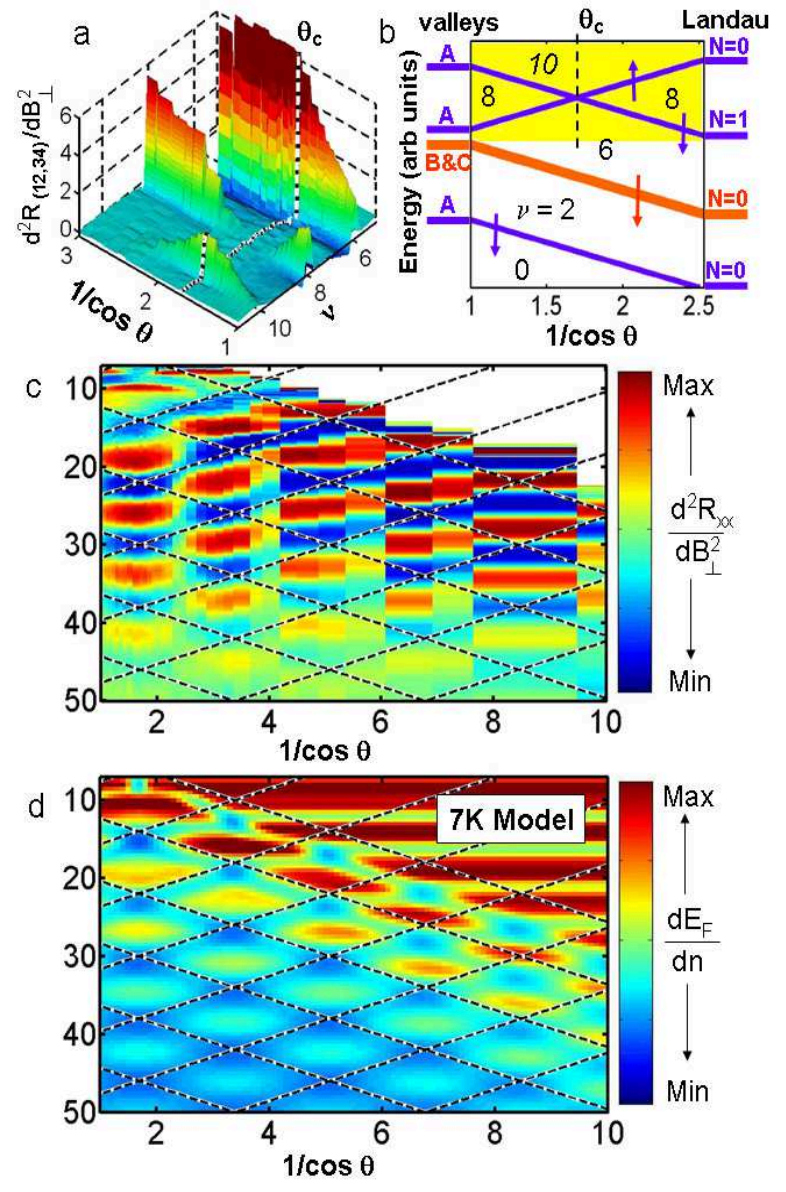

FIG. 3: Data and calculations represented at $n=6.5 \times$ $10^{11} \mathrm{~cm}^{-2}$ and $T=300 \mathrm{mK}$. For the color scales represented in (a), (c) and, (d), a maximum in $d^{2} R_{x x} / d B_{\perp}^{2}$ or $d E_{F} / d n$ corresponds to a minimum in $R_{x x}$. (a) A surface plot of $d^{2} R_{(12,34)} / d \nu^{2}$ versus $1 / \cos \theta$ for filling factors within $5 \leq \nu \leq 11$. At $\theta_{c}$, the $R_{x x}$ minimum at $\nu=8$ disappears while a minimum at $\nu=10$ is at a peak. (b) Schematic energy level diagram following the $7 \mathrm{~K}$ model predicting the two-fold energy levels from the A valleys (spin up and spin down) crossing one another at $\theta_{c}$. (c) A plot of $d^{2} R_{(12,34)} / d B_{\perp}^{2}$ versus $\nu$ and $1 / \cos \theta$. The overlaid schematic fan diagram (dashed lines) represents expectations from the $7 \mathrm{~K}$ model with $\Delta \nu \simeq 8$. (d) A plot of a calculated $d E_{F} / d n$ versus $\nu$ and $1 / \cos \theta$ using the $7 \mathrm{~K}$ model. The same fan diagram used in (c) is overlaid on top of calculations.

spins will overlap with one another. The angles at which these coincidences occur can be observed through the disappearance of minima in $R_{x x}$. One such coincidence is shown in Fig. 3a, where a minimum in $d^{2} R_{(12,34)} / d B_{\perp}^{2}$ corresponds to the disappearance of the $R_{x x}$ minimum at $\nu=8$ and a concomitant maximum occurs at $\nu=10$. Similar behavior is also observed for $d^{2} R_{(13,24)} / d B_{\perp}^{2}$. The second derivatives of $R_{x x}$ are plotted in order to amplify structure and to eliminate the contribution from the positive MR at low $B$ fields. Figure $3 \mathrm{~b}$ depicts a schematic energy level diagram following the $7 \mathrm{~K}$ model and using $B_{\perp}$ values centered at $\nu=8$. The $7 \mathrm{~K}$ model identifies this observed behavior with a crossing between the two-fold degenerate A valleys in upper spin level of the lowest LL $(N=0)$ and the lowest spin level of the second LL $(N=1)$. This crossing occurs when $E_{Z}=E_{C}$. Assuming the effective mass equals $m_{\mathcal{D}}=0.358 m_{o}$, we obtain an enhanced $g^{*}=3.3$ (bare value $g=2$ ). When these parameters are used, the $7 \mathrm{~K}$ model can explain the observed strengthening of the $\nu=6$ minimum with increasing $\theta$ prior to the level crossing and, although not shown, explains why $\nu=12$ is never observed.

The $7 \mathrm{~K}$ model with $m_{\mathcal{D}}=0.358 m_{o}$, implies $\sim 50 \%$ of the electrons are occupying the lowest two valleys at small $B$ fields. If only these contributed to the low $B$ SdH oscillations, a periodicity of $\Delta \nu \simeq 8$ would be expected if the degeneracy due to spin and valley pairs with opposite $\mathbf{k}$ remain unresolved. Figure 3c shows the remainder of the coincidence measurements, where $d^{2} R_{(12,34)} / d B_{\perp}^{2}$ is plotted versus $1 / \cos \theta$ (x-axis) and $\nu$ (y-axis). The plot is a compilation of $R_{(12,34)}$ measurements oriented at 45 different $\theta$ 's and evidence of up to $\sim 5$ level crossings is apparent. A schematic energy fan diagram in accordance with expectations from the $7 \mathrm{~K}$ model, $\Delta \nu=8$ and $g^{*}=3.3$, is overlaid on top of the data, and the intersections of the levels coincides with minima in $d^{2} R_{(12,34)} / d B_{\perp}^{2}$ or maxima in $R_{x x}$. Figure $3 \mathrm{~d}$ shows calculations derived from the $7 \mathrm{~K}$ model of the derivative of the Fermi energy with respect to the density, $d E_{F} / d n$, in the same range plotted in Figure 3c. The same fan diagram used in Figure $3 \mathrm{c}$ is also overlaid on top of the calculations. By applying different Dingle temperatures, $0.6 \mathrm{~K}$ and $2 \mathrm{~K}$, to the carriers originating from the two-fold and four-fold valleys respectively, the $7 \mathrm{~K}$ model is able to reproduce the unresolved spin degeneracy and the absence of oscillations from the higher energy valleys seen in the data. Differing scattering times are commonly observed in two component electron systems with different densities [15].

A likely origin for breaking the valley degeneracy in our 2DES is the miscut of the wafer. This miscut angle, $\psi$, can be derived from the width of the atomic steps on a $\mathrm{H}-\mathrm{Si}(111)$ surface. Figure $1 \mathrm{~b}$ shows an atomic force microscope (AFM) image of a $\mathrm{H}-\mathrm{Si}(111)$ surface obtained from an adjacent piece of the $\mathrm{Si}(111)$ wafer used to fabricate the device. From the AFM image we observe steps

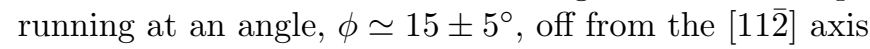
with an average width of $\sim 90 \mathrm{~nm}$. This corresponds to a miscut of $\psi \sim 0.2^{\circ}$ and is approximately about the [11̄0] axis. In the effective mass approximation, this miscut increases the normal mass, $m_{z}$, of the two valleys (A) aligned with the $[11 \overline{2}]$ direction by $\sim 0.5 \%$ relative to the remaining four valleys (B and $\mathrm{C}$ ). As a result, the six-fold symmetry is broken into a 4-2 valley configuration similar to our $7 \mathrm{~K}$ model, but with an energy gap of only $\Delta_{4-2} \sim 2 \mathrm{~K}$. Additionally, the four valleys (B and $\mathrm{C}$ ) occupied at higher energies are split due to the off axis rotation of $\phi$. This energy gap is calculated to 


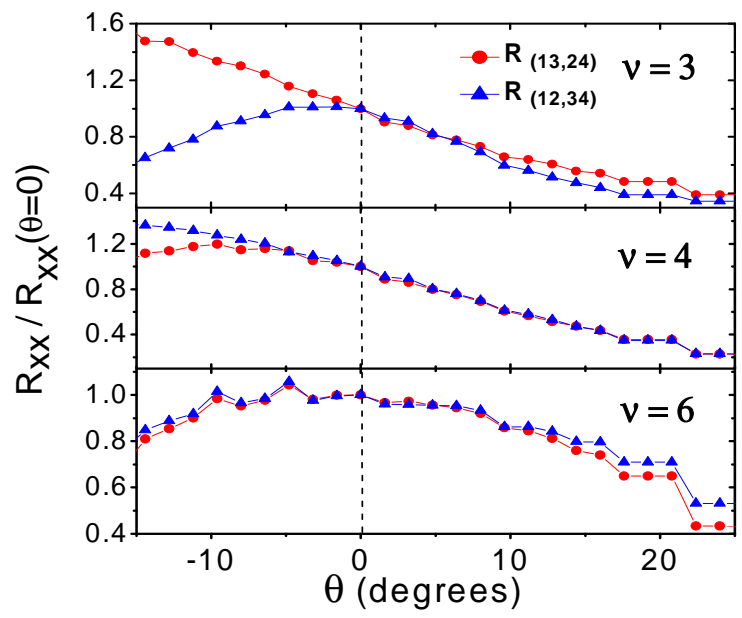

FIG. 4: $R_{x x}$ minima at $\nu=3,4$, and 6 are divided by their respective $R_{x x}$ values at $\theta=0^{\circ}$ and plotted within $-15^{\circ} \leq$ $\theta \leq 25^{\circ}$. Data represents measurements at $T=300 \mathrm{mK}$ and $n=6.5 \times 10^{11} \mathrm{~cm}^{-2}$.

be much smaller, $\Delta_{4} \sim 0.2 \mathrm{~K}$, which is within our experimental uncertainties, but potentially explains the presence of $\nu=4$. It is possible that the discrepancy in the magnitude of $\Delta_{2}$ is due to a many body enhancement. However, these predicted energy gaps do not include possible effects the atomic steps at the surface may have on the confinement of the electronic wavefunction [16].

The simple $7 \mathrm{~K}$ model does break down at high $B$, specifically for the observed minima at $\nu=5,4$, and 3 . Figure 4 displays the behavior of the $R_{x x}$ minima for $\nu=6,4$, and 3 versus small $\theta$. In agreement with the $7 \mathrm{~K}$ model, the $\nu=6$ minimum strengthens as $\sim 1 / \cos \theta$ for small positive and negative tilts about $\theta=0^{\circ}$. However, the $R_{x x}$ minima for $\nu=4$ are observed to have a linear response to small tilts about $B_{\perp}$. For surface electrons which have non-zero off diagonal terms normal to the surface in the effective mass tensor, it is possible to have confinement energies depending linearly on $\theta[17]$. The magnitude of this effect is $\propto \sin \theta \cos \theta$ and has a peak value of $\sim 1.5 \mathrm{~K}$ for $\nu=4$. This agrees well with the observed linear response around small $\theta$ for $\nu=4$.

The observation of $\nu=3$ and 5 indicates that the in-plane symmetry of $\pm \mathbf{k}_{\|}$between opposite valleys is broken on our $\mathrm{H}-\mathrm{Si}(111)$ surface. We also observe $\nu=3$ to be the only state which exhibits anisotropy in $\Delta$ between the two resistance orientations (Table 1). Figure 4 shows that this anisotropy is also dependent on the measurement orientation with respect to $B_{\|}$. This splitting cannot be explained by uniform strain or misorientation effects in the effective mass approximation. It also cannot be easily interpreted using theories developed for valley splittings on [100] Si surfaces [8, 16], where the confinement potential couples valleys with minima in the direction normal to the surface. It is possible that disorder on the surface couples the opposite $\mathbf{k}_{\|}$valleys in our samples. However, anisotropies of $R_{x x}$ developing at low temperatures are also observed in other high mobility $2 \mathrm{D}$ systems [18, 19] and have been attributed to correlated electron phases. The physics may also be similar to the lifting of the sublattice symmetry recently observed in graphene [20], or to skyrmion states in multivalley electron systems 21, 22].

We note that our sample fabrication process is not yet optimized and that prospects for substantially improved mobilities are good. Our results imply that the valley splitting on the $\mathrm{Si}(111)$ surfaces can be tuned by wafer orientation and in-plane magnetic fields. Thus, Hpassivated Si surfaces can be prepared with the maximum number of valley degrees of freedom in which to explore potentially new many-body quantum phenomena, such as exotic fractional quantum Hall states [23], and 'valley singlet' states which are potentially relevant for topological quantum computing [24]. Lastly, the relative ease of incorporating surface engineering within the fabrication process may enable future experiments in which high mobility electrons are coupled to molecules or atoms on an atomically clean semiconductor surface.

Funding was provided by the National Security Agency.

[1] F. Schäffler, Semicond. Sci. Technol. 12, 1515 (1997).

[2] M. L. Lee et al., Thin Solid Films. 508, 136 (2006).

[3] K. Eng, R. N. McFarland and B. E. Kane, Appl. Phys. Lett. 87, 052106 (2005).

[4] G. S. Higashi et al., Appl. Phys. Lett. 56, 656 (1990).

[5] E. Yablonovitch et al., Phys. Rev. Lett. 57, 249 (1986).

[6] M. Hines, Annu. Rev. Phys. Chem. 54, 29 (2003).

[7] J. L. O'Brien et al., Phys. Rev. B 64, 161401(R) (2001).

[8] T. Ando, A. B. Fowler and F. Stern, Rev. Mod. Phys. 54, 437 (1982)

[9] D. C. Tsui and G. Kaminsky, Solid State Comm. 20, 93 (1976).

[10] G. Dorda, I. Eisele and H. Gesch, Phys. Rev. B 17, 1785 (1978).

[11] D. C. Tsui and G. Kaminsky, Phys. Rev. Lett. 42, 595 (1979).

[12] T. Cole and B. D. McCombe, Phys. Rev. B 29, 3180 (1984).

[13] M. J. Kelly and L. M. Falicov, Phys. Rev. Lett. 37, 1021 (1976).

[14] R. A. Smith, Semiconductors, (Cambridge University Press 1978), chap. 5.

[15] H. V. Houten et al., Phys. Rev. B 37 2756(R) (1988).

[16] M. Friesen, M. A. Eriksson and S. N. Coppersmith, Appl. Phys. Lett. 89, 202106 (2006).

[17] F. Stern and W. E. Howard, Phys. Rev. 163, 816 (1967).

[18] M. P. Lilly et al., Phys. Rev. Lett. 82, 394 (1999).

[19] W. Pan et al., Phys. Rev. B 64, 121305(R) (2001).

[20] Y. Zhang et al., Phys. Rev. Lett. 96, 136806 (2006).

[21] M. Shayegan et al., Phys. stat. sol. (b) 243, 3629 (2006).

[22] D. P. Arovas, A. Karlhede and D. Lilliehöök, Phys. Rev. B 59, 13147 (1999).

[23] K. Lai et al., Phys. Rev. Lett. 93, 156805 (2004).

[24] E. Ardonne and K. Schoutens, Annals of Phys. 322, 201 (2007). 